\title{
Holism and Structuralism in $U(1)$ Gauge Theory
}

\author{
Holger Lyre \\ Philosophy Department \\ University of Bonn ${ }^{2}$
}

July 2004

\begin{abstract}
After decades of neglect philosophers of physics have discovered gauge theories - arguably the paradigm of modern field physics - as a genuine topic for foundational and philosophical research. Incidentally, in the last couple of years interest from the philosophy of physics in structural realism-in the eyes of its proponents the best suited realist position towards modern physics - has also raised. This paper tries to connect both topics and aims to show that structural realism gains further credence from an ontological analysis of gauge theories - in particular $U(1)$ gauge theory. In the first part of the paper the framework of fiber bundle gauge theories is briefly presented and the interpretation of local gauge symmetry will be examined. In the second part, an ontological underdetermination of gauge theories is carved out by considering the various kinds of non-locality involved in such typical effects as the Aharonov-Bohm effect. The analysis shows that the peculiar form of non-separability figuring in gauge theories is a variant of spatiotemporal holism and can be distinguished from quantum theoretic holism. In the last part of the paper the arguments for a gauge theoretic support of structural realism are laid out and discussed.
\end{abstract}

Keywords: Gauge theories, gauge symmetry, ontological underdetermination, holism, non-separability, structural realism

\footnotetext{
${ }^{1}$ To appear in Studies in History and Philosophy of Modern Physics 35(4), 2004.

${ }^{2}$ Address: Am Hof 1, D-53113 Bonn, Germany, E-mail: lyre@uni-bonn.de
} 


\section{The interpretation of gauge symmetry}

The house of gauge theories and, accordingly, the house of the philosophical foundations of gauge theories as well, has two wings, occupied by those who advocate either the Hamiltonian or the Lagrangian formalism to represent gauge theories. Among philosophers of physics we find John Earman as one of the strongest supporters of the Hamiltonian view (cf. his 2003, see also Belot and Earman 2001 and Belot 2003). Proponents of the Hamiltonian view consider gauge theories primarily as constraint systems with redundant gauge degrees of freedom. As Henneaux and Teitelboim (1992) put it:

"Physical theories of fundamental significance tend to be gauge theories. These are theories in which the physical system being dealt with is described by more variables than there are physically independent degrees of freedom. The physically meaningful degrees of freedom then reemerge as being those invariant under a transformation connecting the variables (gauge transformation). Thus, one introduces extra variables to make the description more transparent and brings in at the same time a gauge symmetry to extract the physically relevant content. It is a remarkable occurrence that the road to progress has invariably been toward enlarging the number of variables and introducing a more powerful symmetry rather than conversely aiming at reducing the number of variables and eliminating the symmetry."

Supporters of the Lagrangian view, on the other hand, have a quite different notion of what a gauge theory is. Here's a claim from a prominent voice, Andrzej Trautman (1980), that captures the Lagrangian perspective:

"For me, a gauge theory is any physical theory of a dynamic variable which, at the classical level, may be identified with a connection on a principal bundle. The structure group $G$ of the bundle $\mathbb{P}$ is the group of gauge transformations of the first kind; the group $\mathcal{G}$ of gauge transformations of the second kind may be identified with a subgroup of the group $\mathcal{A} u t(\mathbb{P})$ of all automorphisms of $\mathbb{P}$."

This is obviously a quite different conception compared to the former one: the Lagrangian view stresses in particular the importance of an underlying fiber bundle structure to characterize gauge theories. It is, practically speaking, by far the most common and widespread formalism in which gauge theories are presented today.

In this paper I shall adopt the Lagrangian view as well. Some results of the conceptual analysis in this section will perhaps justify this choice. It should however be clear that, since from the mathematical point of view Lagrangian and Hamiltonian formalisms are equivalent, in the end both views must be conceptually intertranslatable. 


\subsection{Gauge principle - the received view}

In his comprehensive collection of the milestone papers of gauge theory in the 20th century, Lochlainn O'Raifeartaigh speaks of the gauge principle as the "cornerstone of modern physics" (O'Raifeartaigh 1995). ${ }^{3}$ The gauge principle indeed serves as the conceptual starting point of many gauge theoretic considerations and it shall be used here in the same spirit. The general idea is to start from a free matter field theory, a Dirac field, say. The Dirac Lagrangian ${ }^{4}$

$$
\mathcal{L}_{D}=\bar{\psi}\left(i \gamma^{\mu} \partial_{\mu}-m\right) \psi
$$

admits the symmetry transformations

$$
\psi^{\prime}=e^{i q \alpha} \psi,
$$

which form the unitary group $U(1)$. As is well known, symmetries in physics do quite generally reflect the invariance properties of certain classes of physical objects. Emmy Noether's famous first theorem is the mathematically precise expression of this factit states that the existence of a $k$-dimensional symmetry group is connected with the existence of $k$ conserved currents. The Noether current of the Dirac field is usually written as the charge density current $\jmath^{\mu}=q \bar{\psi} \gamma^{\mu} \psi$.

There is, on the other hand, electromagnetism, the theory governed by the Maxwell equations and hence the Lagrangian $\mathcal{L}_{M}+\mathcal{L}_{\text {coup }}=-\frac{1}{4} F_{\mu \nu} F^{\mu \nu}-\jmath_{\mu} A^{\mu}$ (for $\mathcal{L}_{\text {coup }}=0$ we get the homogeneous Maxwell equations). $F_{\mu \nu}$, the electromagnetic field strength tensor, can be derived from the potentials via $F_{\mu \nu}=\partial_{\mu} A_{\nu}-\partial_{\nu} A_{\mu}$. In Maxwell's theory the potentials do not play a physical role - the theory just allows for a certain gauge freedom expressed by the gauge transformations

$$
A_{\mu}(x) \rightarrow A_{\mu}^{\prime}(x)=A_{\mu}(x)-\partial_{\mu} \alpha(x)
$$

with an arbitrary scalar function $\alpha(x)$. The transformations (3) are spacetime-dependent and therefore Noether's second theorem applies, which in this case states the existence of the Bianchi identity $\epsilon^{\mu \nu \rho \sigma} \partial_{\nu} F_{\rho \sigma}=0$ (the homogeneous group of the Maxwell equations). ${ }^{5}$

So far, Dirac's and Maxwell's theories are in no relation to each other - two different worlds governed by two different Lagrangians and two different types of equations. However, it turns out that under the replacement

$$
\partial_{\mu} \rightarrow D_{\mu}=\partial_{\mu}+i q A_{\mu}
$$

with $A_{\mu}(x)=-\partial_{\mu} \alpha(x)$ in $\mathcal{L}_{D}$ - known as minimal substitution from classical mechanicsDirac's theory also becomes covariant ${ }^{6}$ under a group of spacetime-dependent transfor-

\footnotetext{
${ }^{3}$ For an historical overview the reader may also consult Cao (1997).

${ }^{4} c=\hbar=1$ throughout the paper.

${ }^{5}$ The importance of Noether's work in variational theory and her corresponding theorems have recently been in the focus of foundational and historical considerations mainly by the work of Brading and Brown (2003) and Earman (2002, 2003).

${ }^{6}$ Here the notion of covariance is used in the sense of form invariance, i.e. a theory is covariant under a certain group of transformations, if the equations of the theory remain unchanged under the transformations.
} 
mations

$$
\psi^{\prime}(x)=e^{i q \alpha(x)} \psi(x) .
$$

Written in terms of the covariant derivative (4), the Dirac Lagrangian (1) must hence be replaced by $\mathcal{L}_{D} \rightarrow \mathcal{L}_{D}+\mathcal{L}_{\text {coup }}$ to restore the local symmetry (5).

Now the logic of the gauge principle, as usually presented, is the idea that the demand of local gauge covariance of $\mathcal{L}_{D}$ in and of itself already leads to the coupling of the Dirac field to an interaction field - in this case to the electromagnetic field. This may be called the received view of the gauge principle. The reason for the received view is that the vector field $A_{\mu}$ in (4), which arises in order to restore the symmetry of the Dirac Lagrangian $\mathcal{L}_{D}$ under local gauge transformations (5), is simply identified with the gauge potential of a non-vanishing gauge field $F_{\mu \nu}$. The problems and inconsistencies with this view will be scrutinized in section 1.3.

Finally, we obtain the total Lagrangian

$$
\mathcal{L}_{D M}=\mathcal{L}_{D}+\mathcal{L}_{\text {coup }}+\mathcal{L}_{M}=\bar{\psi}\left(i \gamma^{\mu} \partial_{\mu}-m\right) \psi-\jmath_{\mu} A^{\mu}-\frac{1}{4} F_{\mu \nu} F^{\mu \nu}
$$

for a proper gauge field theory, i.e. a combined matter field and interaction field theory such as the Dirac-Maxwell theory.

\subsection{Interlude: gauge theories and fiber bundles}

Presented this way, the structure of a gauge theory is apparently based on the interplay between three different types of gauge symmetry transformations:

1. Rigid transformations, usually called global gauge transformations (2),

2. Local gauge transformations, further classified as (following Pauli's 1941 classic): ${ }^{7}$

(a) Gauge transformations of the first kind (5),

(b) Gauge transformations of the second kind (3).

As already mentioned, the concepts involved in gauge theories can suitably be interpreted within the enlarged geometrical arena of principal fiber bundles and their associated vector bundles. ${ }^{8}$

A fiber bundle is a structure $\langle\mathbb{E}, \mathcal{M}, \pi, \mathbb{F}, G\rangle$ with bundle space $\mathbb{E}$, base manifold $\mathcal{M}$, projection map $\pi: \mathbb{E} \rightarrow \mathcal{M}$, fiber spaces $\mathbb{F}_{p}$, and structure group $G$. Fiber bundles can be considered as generalizations of the Cartesian product in the sense that they in general

\footnotetext{
${ }^{7}$ Unfortunately, this terminology is not uniform. Some authors already call global and local gauge transformations transformations of the first and second kind (as Trautmann's quote for instance shows).

${ }^{8}$ Cf. Schutz (1980) or Nakahara (1990) for mathematical expositions of fiber bundles. In the philosophical literature Sunny Auyang's (1995) was the first broad portrayal of fiber bundle theories within the Lagrangian view, but mention should also be made of Cao (1997) and, more recently, Healey (2001) and Nounou (2003).
} 
only locally look like $\mathcal{M} \times \mathbb{F}$ (here we assume all fibers $\mathbb{F}_{p}$ at $p \in \mathcal{M}$ to be homeomorphic to the typical fiber $\mathbb{F}$ ). A local trivialisation is then given by a diffeomorphic map $\phi_{i}: \mathcal{U}_{i} \times$ $\mathbb{F} \rightarrow \pi^{-1}\left(\mathcal{U}_{i}\right)$ within some open set $\mathcal{U}_{i} \subset \mathcal{M}$. In order to obtain the global bundle structure the local charts $\phi_{i}$ must be glued together with transition functions $t_{i j}(p)=\left(\phi^{-1} \circ \phi_{j}\right)(p)$. If all transition functions can be taken to be the identity the bundle is called trivial. In this case not only its local but also its global structure is that of $\mathcal{M} \times \mathbb{F}$.

In physics two classes of bundles play a crucial role: If the fiber is given by some $n$ dimensional linear vector space $\mathbb{V}^{n}$ the bundle is called a vector bundle $\mathbb{E}\left(\mathcal{M}, \mathbb{V}^{n}, G L(n, \mathbb{V})\right)$. The general structure group for $\mathbb{V}^{n}=\mathbb{R}^{n}$ is $G=G L(n, \mathbb{R})$. For a principal bundle $\mathbb{P}(\mathcal{M}, G)$ the fiber $\mathbb{F}$ is identical to the structure group $G$. To any principal bundle there naturally exists an associated vector bundle with the same structure group and transition functions.

Four types of bundles can in general be distinguished:

1. trivial bundles with flat connections

2. trivial bundles with non-flat connections

3. non-trivial bundles with flat connections

4. non-trivial bundles with non-flat connections

Cartesian product spaces can be considered as bundles of the first type with Galileian spacetime as a concrete physical example. It has absolute time $\mathbb{R}$ - the bundle's base space - and relative space $\mathbb{R}^{3}$, i.e. fibers with structure group $O(3)$. The bundle is globally isomorphic to the direct product $\mathbb{R} \times \mathbb{R}^{3}$ and therefore trivial. Physically speaking, the flatness of the connections indicates that space is non-dynamical. Now suppose space or rather spacetime to be curved according to some dynamics as in general relativity. Suppose further that the spacetime base manifold is topologically trivial, then this gives an example of the second case, since one can prove that any bundle over a contractible base space is a trivial bundle. Of much more interest are of course the non-trivial cases. A simple example is the line bundle over a circle, i.e. a structure which locally looks like $\mathbb{S}^{1} \times \mathbb{I}$ with $\left.\left.\mathbb{I}=\right] 0,1\right]$, and which can globally either be a cylinder or a Möbius strip. In this latter case the structure group is $\mathbb{Z}_{2}=\{\mathbb{1},-\mathbb{1}\}$. Both cylinder and Möbius strip are however locally isomorphic to the flat plane, the bundle is therefore considered a bundle with "flat connections" between the fibers, that is, without curvature. An example for the fourth, most general case, would finally be given by a curved Riemannian space with some globally non-trivial topology.

In the Lagrangian view of gauge theories one usually considers fiber bundles over spacetime $\mathcal{M}$ as base space with a continuous Lie group, the gauge group $G$, as structure group. The connection of the principal bundle $\mathbb{P}(\mathcal{M}, G)$ is physically interpreted as a gauge potential. Mathematically, a connection can be thought of as a rule which decomposes the tangent of $\mathbb{P}$ into a horizontal and a vertical part $\mathbb{T}_{u} \mathbb{P}=\mathbb{V} u \mathbb{P} \oplus \mathbb{H}_{u} \mathbb{P}$ for every $u \in \mathbb{P}$. Let $\mathfrak{g}$ be the Lie algebra of $G$, then the connection is defined as a $\mathfrak{g}$-valued 
one-form projecting $\mathbb{T}_{u} \mathbb{P}$ to $\mathbb{V}_{u} \mathbb{P} \cong \mathfrak{g}$. Physicists are familiar with this concept as the covariant derivative (4) already introduced. ${ }^{9}$ Furthermore, matter fields are defined as local sections in the associated vector bundle $\mathbb{E}$ of $\mathbb{P}$. A bundle section is defined as a mapping $\sigma: \mathcal{M} \rightarrow \mathbb{E}$ and can be thought of as a generalization of a tangent vector field. With $\pi(\sigma(p))=p$ the section $\sigma(p) \in \mathbb{F}_{p}$ is local (conversely, a bundle is called trivial, if it admits a global section).

We see that in bundle terminology the distinction between local gauge transformations of the first kind - matter fields as sections in $\mathbb{E}$ - and second kind - gauge potentials as $\mathbb{P}$ connections - is reflected by the distinction between $\mathbb{E}$ and $\mathbb{P}$. The generators of the gauge group $G$ represent gauge bosons, the bundle curvature - the covariant derivative of the connection and, hence, a $\mathfrak{g}$-valued two-form - encodes the interaction field strength. In this terminology the bundle structure of the Dirac-Maxwell theory - and QED, respectivelyis $\mathbb{P}\left(\mathbb{R}^{1,3}, U(1)\right)$ with Minkowski spacetime $\mathbb{R}^{1,3}$. Yang and Mills in 1954 extended this approach to higher unitary and, thus, non-abelian gauge groups $S U(n)$, two years later Utiyama was the first (published!) approach considering gravitational gauge theories. ${ }^{10}$

\subsection{Are gauge transformations observable? - To overcome the received view of the gauge principle}

The received view of the gauge principle is widely adopted, as the following quotes from textbooks show. First Aitchison and Hey (1982, p. 176):

"We shall demonstrate that such a phase invariance is not possible for a free theory, but rather requires an interacting theory ... The demand of this type of phase invariance will then have dictated the form of the interaction-this is the basis of the gauge principle."

An even stronger claim can be found in Ryder (1996, p. 79):

"Present day theories ... state that interactions between fundamental fields (like electrons, quarks, weak vector bosons, and so on) are dictated by a gauge principle. ...it emerges that, in order to have a local symmetry, we need a spin 1 massless gauge field, whose interaction with the 'matter' fields is dictated uniquely."

From these quotes one may get the impression that the application of the gauge principle - the demand that the theory is invariant under local gauge transformationsin one way or the other changes physics. But this would leave us with a puzzle -quite aptly noticed by Michael Redhead $(1998$, p. 503) in his truly justified question: “... how

\footnotetext{
${ }^{9}$ Different, yet equivalent definitions of bundle connections and their close relation to the physicist's understanding are discussed in Drechsler and Mayer (1977).

${ }^{10}$ For the history see again O'Raifeartaigh (1995) and Cao (1997). For philosophical issues concerning gauge theories of gravity the reader may consult Liu (2001) and Lyre and Eynck (2003).
} 


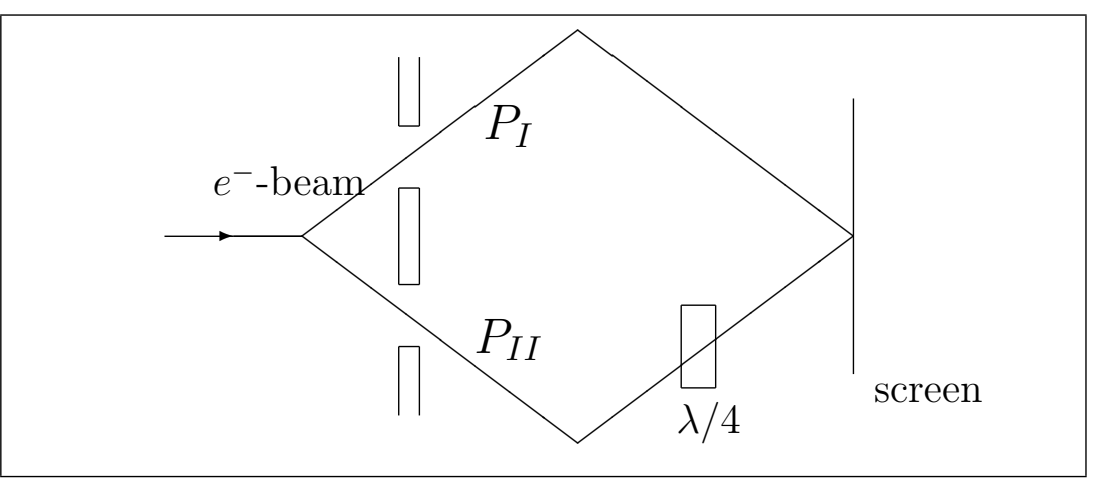

Figure 1: Schematic configuration of the 't Hooft (1980) experiment.

can symmetry under a mere choice of conventional representation dictate any genuinely physical principle at all?"

In order to scrutinize this puzzle, our first concern should be the question, whether gauge transformations may yield any observable effects (as recently stated by Kosso 2000, for instance). Let us first consider global gauge transformations. In the case of quantum gauge theories - the case of interest here - gauge transformations are transformations of the phase of the wave function $\psi \rightarrow \psi e^{i \chi}$. Now the question, whether global phase transformations are observable, can safely be answered in the negative, since changes in the global phase - a rotation of the entire Hilbert space - do not change any observables in quantum theory. More precisely, the expectation value $\langle\hat{A}\rangle=\frac{\langle\psi|\hat{A}| \psi\rangle}{\langle\psi \mid \psi\rangle}$ of any observable $\hat{A}$ is invariant under $\psi \rightarrow \psi e^{i \chi}$. Global phase transformations are therefore clearly without observable effects. But what about local phase transformations $\psi(x) \rightarrow \psi^{\prime}(x)=\psi(x) e^{i \chi(x)}$ ?

Gerard 't Hooft (1980) offers a seemingly convincing argument to support the observability of local phase transformations (and, in turn, the received view of the gauge principle). He considers a simple double-split experiment with an interference pattern. By inserting a phase shifter behind the slit in one of the two paths a shift of the interference pattern occurs (the schematic experimental configuration is given in figure 1). The very point now is that, according to 't Hooft, the phase shifter should be considered as a realization of a local phase transformation. However, applying a phase shift only to one part of a total wave function which is in a superposition of many parts - such as the double slit wave function $\psi=\psi_{I}+\psi_{I I}$ on the two paths $P_{I}$ and $P_{I I}$-leads to a relative phase shift instead of a proper phase transformation. As Brading and Brown (forthcoming) quite correctly put it: "Local gauge freedom is the freedom to vary the overall phase of the wavefunction from point to point, but it is not the freedom to vary the phase of $\psi_{I}$ with respect to $\psi_{I I}$ at a single spacetime point. Under a local gauge transformation the phase of $\psi_{I}$ at some point on the screen will be changed by the same amount as the phase of $\psi_{I I}$ at that same point. What we need in order to change the interference pattern is a relative phase transformation of $\psi_{I}$ with respect to $\psi_{I I}$ at each point on the screen." For instance, a $\lambda / 4$-phase shifter in $P_{I I}$ leads to $\psi_{I I} \rightarrow \psi_{I I} e^{i \lambda / 4}$. This changes the total wave function 
$\psi$ to $\tilde{\psi}=\psi_{I}+\psi_{I I} e^{i \lambda / 4}$ and, quite obviously, $\psi$ and $\tilde{\psi}$ do have different expectation values. For that reason a clear distinction must be made between relative phase shifts and local phase transformations - or more generally between transformations of subsystems of a total system and symmetry transformations proper. 't Hooft's experiment only shows the observability of the former.

There is yet another argument supposedly in favor of an observability interpretation of local phase transformations: The eigenvalue equation of the momentum operator $\hat{p}_{\mu}=$ $-i \partial_{\mu}$ for a plane wave $\psi=e^{i p x}$ is simply given by $\hat{p}_{\mu} \psi=p \psi$. Apparently, the locally phase transformed wave function now obeys the new equation $\hat{p}_{\mu} \psi^{\prime}=\left[p+\partial_{\mu} \chi(x)\right] \psi^{\prime}$. The argument is not really different from the gauge argument itself, simply because the inhomogeneous term in the covariant derivative is indeed nothing but the gradient of the local phase $q A_{\mu}(x)=-q \partial_{\mu} \alpha(x)=-\partial_{\mu} \chi(x)$-and we shall see now that this "change" is not observable.

Let us, for the sake of clarity, consider the general form of local gauge transformations of the first kind $\psi^{\prime}=e^{i \chi(x)} \psi$ and hence a covariant derivative

$$
D_{\mu}=\partial_{\mu}-i \partial_{\mu} \chi(x)
$$

instead of (4). To see in more general terms that the Lagrangians $\mathcal{L}_{D}$ and $\mathcal{L}_{D}^{\prime}=$ $\bar{\psi}^{\prime}\left(i \gamma^{\mu} D_{\mu}-m\right) \psi^{\prime}$ are indeed equivalent, the following consideration should suffice: Let $|x\rangle$ be the position representation of a wave function $\Psi(x)=\langle x \mid \phi\rangle$, where $\{|\phi\rangle\}$ span an abstract Hilbert space, then local gauge transformations $\left|x^{\prime}\right\rangle=e^{i \chi(x)}|x\rangle=\hat{U}|x\rangle$ must properly be seen as mere changes in $|x\rangle$. Such a change of the representation now obviously affects the operators as well. Generally, Hilbert space operators transform as $\hat{O}^{\prime}=\hat{U} \hat{O} \hat{U}^{+}$, and in the particular case of the derivative (or the momentum operator, respectively) we get the covariant derivative (7), which is thus uncovered as nothing but a mere change in the position representation.

Therefore, by using the appropriate momentum operator $\hat{p}_{\mu}^{\prime}$ in the new representation, the inhomogeneous term in the expectation value of the original momentum operator, as introduced in the preceding section, is cancelled and, hence, from $\hat{p}_{\mu}^{\prime} \psi^{\prime}=p \psi^{\prime}$ it can easily be seen that no physical change has occurred. Thus, local phase transformations turn out as not observable at all. Furthermore, since the tacit substitution $\partial_{\mu} \chi=q A_{\mu}$ used in the gauge principle has of course no physical meaning, the true Noether current of the Dirac theory is really just the probability density current $S^{\mu}=\bar{\psi} \gamma^{\mu} \psi$. The essential point of all this is that local phase transformations mean no more and no less than simple changes in the representation - at best be interpreted as changes in the generalized fiber bundle coordinates and much on the same - dynamically meaningless! - footing of ordinary coordinate transformations in spacetime. ${ }^{11}$ What does all this tell us now about our understanding of the gauge principle?

\footnotetext{
${ }^{11}$ Accordingly, this result generalizes to the case of gauge transformations in gauge theories of gravity as well. Roughly stated, local gauge transformations in this case are local Lorentz rotations or Poincaré translations of tetrads $\theta_{\mu}^{\alpha}(x)$ representing local reference frames. At first glance it looks as if such transformations would be observable, however, because of $g_{\mu \nu}(x)=g_{\alpha \beta} \theta_{\mu}^{\alpha}(x) \theta_{\nu}^{\beta}(x)$, any transformation of the tetrads leads to a recalibration of the metric as well leaving the total physical state unchanged.
} 
Clearly, the question whether some transformations are observable or whether the dynamical laws are invariant under those transformations are, in the first place, two separate issues. However the logic of the received view of the gauge principle suggests to connect these two points. As we have seen in section 1.1, the demand of local gauge invariance of a formerly interaction-free Lagrangian leads to a covariant derivative where the inhomogeneous term is interpreted as indicating the existence of a non-vanishing and observable interaction field. The message of the gauge principle is therefore often put as if the (supposedly observable) effect of local gauge transformations would be compensated by introducing an appropriate (seemingly observable) gauge interaction field. 't Hooft makes the same claim by pointing out that the shift of an interference patterns in a double slit experiment can either be produced by asserting a phase shifter or by asserting a magnet behind the slit, so that an Aharonov-Bohm experiment is realized. He then concludes that "the magnetic field compensates the phase shift" and that "in gauge theories fields are introduced to restore the symmetry."

We will address the Aharonov-Bohm experiment in great detail in section 2, but for the moment we just record the fact that 't Hooft's claim doesn't hold because local phase transformations are already unmasked as not observable. From this insight, however, the whole logic of the received view breaks down. Since the introduction of an interaction field as intended by the received view seemingly changes physics (those fields are even directly observable themselves), it is necessary from this view to consider local gauge transformations as changing physics as well in order to tell the story about compensation. Since, however, local gauge transformations can be shown as not observable, the received view proves itself untenable.

Hence, the way the gauge principle is used in a considerable part of the physics literature - such as Ryder - is certainly overstated. For a mere change of representation cannot "dictate" a real physical interaction. The demand of local gauge invariance of the Dirac Lagrangian (1) under a certain group $G$ leads to the replacement (4) of the usual by the covariant derivative, where an extraneous inhomogeneous connection term occurs. The mathematical formalism ensures that this will be a $G$-connection. From the pure logic of the gauge argument, however, this connection is still flat, i.e. with vanishing bundle curvature, whereas in an overstated reading of the gauge principle it looks as if one could enforce the existence of a non-vanishing curvature tensor, i.e. a real dynamical field. ${ }^{12}$

\footnotetext{
${ }^{12}$ Similar critical readings of the gauge principle have been discussed in the last years by the following authors: Brown (1999), Healey (2001), Lyre (2000, 2001), Martin (2002, 2003), and Teller (2000).-One may however, as indeed Paul Teller in an unpublished draft (1999) contrary to his 2000 publication does, raise the objection that the above analysis of the application of the gauge argument in ordinary quantum wave mechanics fails, since - as differential operators - position and momentum are strictly speaking no linear operators on the Hilbert space $L^{2}(\mathbb{R})$, i.e. they cannot be defined on all vectors of $L^{2}(\mathbb{R})$ but only on a dense subset of differentiable functions thereof. The usual stance to this is a pragmatic one: the gauge argument has either to be applied within the approximation of considering position and momentum as "almost" linear operators or one has to rigorously refine quantum mechanics in terms of rigged Hilbert spaces or other means. By way of contrast, Teller (1999) comes to the astonishing conclusion that the gauge argument only applies in the second quantized formalism and that here the argument indeed leads
} 
Local gauge symmetry can thus simply be restored by writing the theory "generally covariant" by means of a covariant derivative. The situation is indeed entirely analogous to the case of general relativity, where Christoffel symbols in the geodesic equation might simply occur due to choosing "curvilinear" coordinates in flat Minkowski spacetime. The Levi-Civita connection by itself does therefore not necessarily represent a true gravitational potential with a non-vanishing gravitational field, i.e. non-vanishing Riemann curvature as a result of a certain dynamics. Rather, the connection term might just occur due to some peculiar choice of coordinates.

Does this mean then that the gauge principle is physically void? Surely, the general question is what it means for a group of transformations to constitute a symmetry of a given dynamical system. Quantum electrodynamics, for instance, is certainly quite aptly characterized as a $U(1)$ gauge theory. The physical significance of this statement lies in the fact that the dynamical field of the theory, the electromagnetic field strength tensor $F^{\mu \nu}$, is characterized by this symmetry as the curvature tensor of the $U(1)$-connection, and that, hence, the Maxwell Lagrangian $\mathcal{L}_{M}=-\frac{1}{4} F_{\mu \nu} F^{\mu \nu}$ shows invariance under (global and local) $U(1)$ symmetry. So of course the characterization of a gauge theory by some gauge group $G$ as its symmetry group is a physically meaningful statement, insofar as the existence of a real dynamical interaction field shall be indicated. But this very existence has to be tested empirically and cannot be enforced by some symmetry requirement.

Perhaps this is what many physics authors have in mind when they write about the gauge principle - and perhaps Aitchison's and Hey's statement that the demand of local phase invariance dictates the form of the interaction - i.e. the form of the coupling termshould be read this way as well. But then it must be noted that it is not necessary to call this a "principle" since what happens is merely that one plugs in a certain gauge group $G$ in the first place - in the above sense of being an empirically already vindicated symmetry group - and then gets the corresponding $G$-connection as a mere mathematical consequence.

A final remark on some possible conceptual weaknesses of the Hamiltonian view should be made. From the Lagrangian $\mathcal{L}_{D M}(6)$ we can gather what should perhaps be considered a genuine gauge theory: a coupled matter field and interaction field theory (here: the Dirac-Maxwell theory - again: with the gauge principle not being sufficient to introduce $\mathcal{L}_{D M}$ starting from the Dirac theory $\mathcal{L}_{D}$ alone). As opposed to this, sometimes in the literature Maxwell's theory $\mathcal{L}_{M}$ is already called a gauge theory, but perhaps one should in this case rather speak of a theory with a mere gauge freedom. The Hamiltonian formalism seems not to be suited to grasp this subtle distinction, which corresponds to the possibility of distinguishing between first kind local gauge transformations of the matter fields and second kind transformations of the gauge potentials. This is also related to the fact that the Hamiltonian view lacks a genuine fiber bundle picture, where, as already mentioned, this distinction is reflected in the concepts of principal and associated vector

to the existence of a non-flat connection! I am sceptical about this, but a rigorous discussion of this would mean to delve into the quantum field theoretic formalism which is not the purpose of the present paper. 


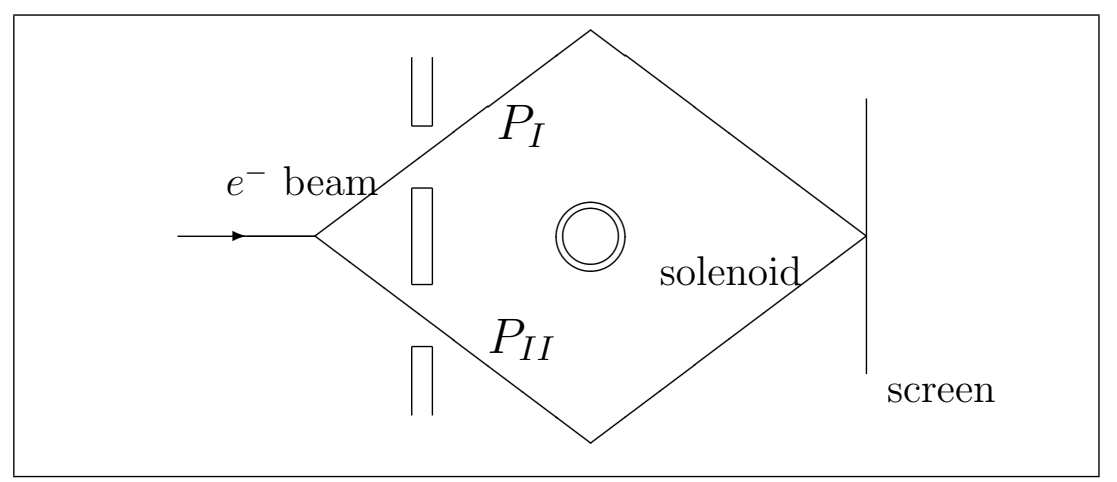

Figure 2: Schematic experimental configuration of the AB effect.

bundles. At the end, of course - given the mathematical equivalence between Lagrange and Hamiltonian view - these weaknesses are perhaps only apparent ones. However from the practising physicist's point of view they make quite a difference.

\section{The ontology of gauge theories - underdetermined}

Aharonov and Bohm (1959) were the first who realized that in quantum gauge theories, already on the non-relativistic and semi-classical level (i.e. the minimal coupling of the Schrödinger wave function to an electromagnetic field), remarkable effects are possibleremarkable in the sense that no entirely "local" explanation can be given. They discussed in their paper what is nowadays called the magnetic and the electric AB effect. ${ }^{13}$

In fact, the $\mathrm{AB}$ effect forms only the prelude to the even more general class of topological effects which are possible in gauge theories with an underlying non-trivial fiber bundle structure and which are known as monopoles or instantons for instance (cf. Nakahara 1990 and Ryder 1996, chap. 10). It should, however, be noticed that the AB effect can finally be distinguished from these entirely topological effects, as will become clear in section 2.3. It is nevertheless sufficient for our interest in the basic ontology of gauge theories to consider the $\mathrm{AB}$ effect as a first example here.

\subsection{The Aharonov-Bohm effect and different notions of locality}

Put crudely, the magnetic AB effect is just this: A split electron beam passing - in a field-free region - around a solenoid which confines a magnetic field nevertheless shows a shift in the interference pattern upon alteration of the magnetic field. A schematic

\footnotetext{
${ }^{13} \mathrm{Cf}$. Peshkin and Tonomura (1989) for a detailed review of the intense discussion in the physics literature the $\mathrm{AB}$ effect has caused in its first three decades of history. In recent times the $\mathrm{AB}$ effect has also attracted interest within the philosophical literature. Here, mention should be made of Batterman (2003), Healey (1997, 1999, 2001), Leeds (1999), Liu (1994), Maudlin (1998), Nounou (2003) as well as Lyre (2001) and Eynck, Lyre and Rummell (forthcoming).
} 
experimental setting of the $\mathrm{AB}$ effect is given in figure 2. Quantitatively, the phase shift can be calculated from the loop integral over the potential, which-due to Stokes' theorem - is related to the magnetic flux

$$
\Delta \chi=q \oint_{\mathcal{C}} \vec{A} d \vec{r}=q \int_{\mathcal{S}} \vec{B} d \vec{s}=q \Phi_{m a g}
$$

The AB effect must indeed be considered as demonstrating a genuine non-local interaction between the magnetic field and the quantum wave function and cannot easily be explained away as a side-effect of some sort of improper shielding. The reason for this is that, on the one hand, the magnetic field can in principle - and as opposed to the schematic experimental setting in figure 2-perfectly be confined in toroidal magnets (cf. Tonomura 1998) and that, on the other hand, the unavoidable penetration of the quantum wave function into the solenoid is not correlated to any scaling of the effect with the quality of the solenoid's shielding. It is therefore obvious that the effect shows at least some sort of non-locality. But which exactly? To tackle this question we must delve into the different meanings the concept of non-locality may have in physics (cf. also Healey 1997 on non-locality and the AB effect).

As argued in Eynck, Lyre and Rummell (forthcoming), one may at least distinguish three different notions of locality in physics (or metaphysics, respectively). The first two of them, local action and point-like interaction, are strongly connected with the very concept of field theories in physics. Local action can be restated as follows:

\section{Local action}

All causes of an event propagate via some continuous physical processes.

Technically this is established by the build-in causal light cone structure of modern field theories. The structure of Lagrangians in field theory and indeed the very idea of the field itself - as a quantity with values at spacetime points - give rise to point-like interaction ${ }^{14}$ as a second important locality concept:

\section{Point-like interaction}

Interacting entities can be defined within arbitrarily small spacetime regions (usually idealized as spacetime points), couple to each other in that regions and are non-zero in overlapping regions.

Now, the paradigm for a violation of locality in physics is of course the case of quantum entanglement. There is widespread belief that this particular sort of non-locality is perhaps best captured as a failure of the separability of quantum systems, which can mathematically be traced back to the particular tensor product structure of the Hilbert

\footnotetext{
${ }^{14}$ The term "point-like" may be misleading here, since (quantum) fields are distributions over spacetime and realistically therefore only properly defined over some dense domain. So point-like interaction may equally well be read as interaction within arbitrarily small spacetime regions (and in this sense local). This - still informal - definition is sufficient for our needs.
} 
state space: in quantum theory, the state vector of a compound system can in general not be factorized into a product of state vectors of the subsystems, but must rather be described as a superposition state.

On the assumption that the state of a system specifies the system's properties, one may grasp the idea of separability equally well in saying that it must be possible to gain the properties of the compound system from the properties of the parts. In more philosophical terms separability is often explained in recourse to the notion of supervenience: properties of the compound system should supervene on the properties of the parts. Different notions of separability can then be gained by considering either relational or intrinsic properties or both in the supervenience basis (cf. Howard 1985, Teller 1986, Healey 1991, Esfeld 2001). An appropriate definition of separability for our aims - without reference to the notion of supervenience - is the following:

\section{Separability}

Given a physical system $S$ and its exhaustive, disjoint decomposition into spatiotemporally divided subsystems, it is possible to retrieve the properties of $S$ from the properties of these subsystems.

Once again, quantum theory violates this concept of separability by the existence of EPR-Bell correlations in entangled states, where the properties of these states cannot be retrieved from the properties of the spacelike separated subsystems.

A further notion, closely related to the failure of separability and sometimes even identified with non-separability is holism: properties of the whole do not supervene on properties of the parts. A fruitful distinction between the two concepts might perhaps be gained by giving up the particular restriction to spatiotemporally defined domains of the system and to generalize to all kinds of - perhaps abstract - domains. In such a way we obtain the following broader definition:

\section{Holism}

Given a physical system $S$ and its exhaustive, disjoint decomposition into subsystems, it is impossible to retrieve the properties of $S$ from the properties of these subsystems.

In this sense non-separability can be understood as a special case of holism tied to spatiotemporal domains, and there are cases in quantum theory where no question of a spatiotemporal separation of the systems is concerned, e.g. cases of spin entanglement of electrons in atoms, where the electrons are not individually localized, or superposition cases of different configurations of certain molecules, or cases of quantum parallelism in quantum computers. Quantum theory does therefore, in this terminology, not only exhibit non-separability, i.e. spatiotemporal holism, but holism in general.

In the following we shall see whether and which of the above notions apply to the $\mathrm{AB}$ effect. As a result of our further discussion it will turn out that it is possible to shift the ontological costs back and forth between different possible interpretations of the 
$\mathrm{AB}$ effect - and this will include different combinations of confirmation and violation of the three locality notions, so that no general judgement about the precise form of nonlocality in the AB effect can be given. From this perspective the question about the origin of non-locality of the $\mathrm{AB}$ effect becomes an interpretation-dependent issue.

\subsection{From $\mathrm{AB}$ to $\mathrm{ABC}$ : Three Interpretations of the $\mathrm{AB}$ effect}

With the above conceptual machinery of different notions of locality, I will now turn to the primary ontological concern of this section: What are the basic entities involved in the AB effect? As we shall see shortly, the answer to this question depends strongly on our metaphysical premisses about locality.

Looking from pure classical electrodynamics, the issue in question seems to have a straightforward answer: only the the field-strengths count as observable entities, the potentials merely play the role of auxiliary functions and are thus to be considered as elements of redundant mathematical surplus structure. One may seek to interpret the $\mathrm{AB}$ effect in the same spirit. What would then be the further consequences of such an interpretation, which only takes the $\vec{B}$-field as real and which might therefore be dubbed the B-interpretation? Obviously, since the $\psi$-field (outside the solenoid) and the $\vec{B}$ field (inside the solenoid) do not exist in overlapping regions, the B-interpretation violates point-like interaction. Moreover, since no continuous physical transmission process from the change of $\vec{B}$ to the shift of the interference pattern exists, it also violates local action, that is, we have a case of action-at-a-distance. The condition of separability, however, is satisfied within the B-interpretation.

Some, but not many in the physics community would take up the B-stance (cf. DeWitt 1962 as an early supporter). Much more common is the view Richard Feynman holds in his famous lectures (1963):

"In our sense then, the A-field is 'real.' You may say: 'But there was a magnetic field.' There was, but remember our original idea-that a field is 'real' if it is what must be specified at the position of the particle in order to get the motion. The $\boldsymbol{B}$-field in the whisker acts at a distance. If we want to describe its influence not as action-at-a-distance, we must use the vector potential."

In sticking to the $\vec{A}$-field as the genuine real entity in gauge theories, the $\mathrm{AB}$ effect gets an A-interpretation instead of the B-interpretation above (giving the acronym AB an entirely new meaning...). The clear advantage of this view, as Feynman has pointed out, is the restored local interaction between $\psi$ and the gauge potential $\vec{A}$, which more precisely means that the A-interpretation conforms to point-like interaction as well as to local action. This advantage, however, comes at a high price: since gauge potentials are gauge-dependent quantities, the values of $\vec{A}$ associated to space regions of any partition are no observable properties. This in turn means that the "real" entities become dependent on our conventional choice of gauge - on, as we saw, mere generalized coordinates! 
Furthermore, one may wonder whether the A-interpretation is separable and can therefore - at least-render the $\mathrm{AB}$ effect as local. But even this is not the case: because of the gauge freedom still inherent in the potentials, a partition into observable properties fails. This is equivalent to the fact that no preferred gauge can be given for the total configuration space of the electron. Therefore, the A-interpretation violates separability.

Remarkably, the AB effect also allows for a third interpretational option. This Cinterpretation considers holonomies (i.e. closed curves $\mathcal{C}$ ) as basic entities. Technically speaking, holonomies are elements of the structure group due to a loop lift: Let $\mathcal{C}:[0,1] \rightarrow$ $\mathcal{M}$ be a parametrized curve in base space, then, generally, the horizontal lift of a closed curve with $\mathcal{C}(0)=\mathcal{C}(1)$ does not guarantee that the horizontal lift $\overline{\mathcal{C}}$ in bundle space is a closed loop as well, but rather $\overline{\mathcal{C}}(1)=\overline{\mathcal{C}}(0) g$ with $g \in G$. As we already saw from $(8)$, the AB phase shift is given by a $U(1)$-holonomy $S(\mathcal{C})=\oint_{\mathcal{C}} A_{\mu} d x^{\mu}$. Since the phase $\chi$ is no single-valued function, one rather uses the Dirac-Wu-Yang phase factor $H(\mathcal{C})=$ $e^{i q S(\mathcal{C})}$. Holonomies are $U(1)$-gauge invariant quantities, they do, however, transform homogeneously under $U(n)$ for $n>1$. The most appropriate, generally gauge invariant formalism takes Wilson loops $\operatorname{tr} H(\mathcal{C})$ as basic entities. It can be shown that, at least on the classical level, the connection and loop formulation are equivalent, and strong indicators exist that all relevant gauge-invariant content of a gauge theory can be reconstructed from the Wilson loops (cf. Loll 1994). Gambini and Pullin (1996) have presented a worked-out and genuine approach for gauge theories within the loop representation.

Besides the advantage of leading directly to gauge invariant and, hence, observable quantities such as the magnetic flux, the C-interpretation also conforms to local action, though it should be noted that the dynamics of loops takes place in an appropriate state space, the loop space, and that its detailed mathematical structure is still a matter of ongoing research (cf. again Loll 1994, and Gambini and Pullin 1996). While, on the one hand, the C-interpretation conforms to observability and local action, it does, on the other hand, violate point-like interaction (in quantum gravity programs the loop approach gives rise to even transcend the traditional picture of spacetime as the basic arena of physics altogether).

Finally we must ask how things stand with the C-interpretation and separability. Indeed the answer depends on how we apply the expression "spatiotemporal support $R$ " in the definition given in section 2.1. As a matter of fact the gauge field strength at a point $p$ can be represented by considering the limit of tinier and tinier loop holonomies around $p$. Due to this equivalence of gauge field strengths and infinitesimal holonomies the holonomy properties of a finite loop enclosing a certain surface $\mathcal{S}$ supervene on the collection of infinitesimal loops around points on that very surface (just because such a collection of infinitesimal holonomies encodes the same physical information as the total collection of field strengths at any point on $\mathcal{S}$ ). This is what Healey (2001) calls the "loop supervenience of holonomy properties" and which would speak in favor of the demand of separability in the C-interpretation by identifying $\mathcal{S}$ with $R$. But in effect this amounts to reducing the $\mathrm{C}$-interpretation to the $\mathrm{B}$-interpretation. It is therefore necessary to consider 


\begin{tabular}{|l|c|c|c|}
\hline & \multicolumn{3}{|c|}{ - interpretation - } \\
& A & B & C \\
\hline point-like interaction & yes & no & no \\
local action & yes & no & yes \\
separability & no & yes & no \\
measurability & no & yes & yes \\
& & & \\
\hline
\end{tabular}

Table 1: "Ontological costs" of the three rivaling interpretations of gauge theories in terms of potentials $(\mathbf{A})$, field strengths $(\mathbf{B})$, or holonomies $(\mathbf{C})$ as their basic entities.

all and only the points as sets $R$ which constitute the particular holonomy loops. On this view the thesis of separability is clearly violated, since now the holonomy properties must be seen as global properties of loops which cannot be retrieved from the intrinsic local properties of any partition of the loops (Healey 2001 comes to the same conclusion). In this sense we must speak of a failure of separability within the context of the C-interpretation.

All of the above amounts to saying that the AB effect in terms of its rivaling A-, B-, C-interpretations seems to provide an intriguing example of theory underdetermination by empirical evidence, since the three interpretations are on the one hand empirically equivalent while on the other hand manifestly different as far as their underlying ontology is concerned. A compact overview can quite neatly be given in the form of table 1 . It shows that, prima facie, the ontological costs of the three interpretations are balanced. At this stage it solely depends on our metaphysical premisses concerning locality and the measurability of the primary entities in our physics whether we prefer one over the other interpretations.

Two further remarks should be made: Firstly, within the philosophical literature the C-interpretation - not only in view of the AB effect but within the context of gauge theories in general - has attracted some interest. Belot (1998) indeed distinguishes the same three interpretations as we do, but as interpretations of classical electromagnetism already, and he considers two notions of locality, synchronic and diachronic, which are related but not identical with our notions of separability and local action. Belot then tries to show that the B-interpretation does not hold in the face of the AB effect and he finally favors the C-interpretation-because of the failure of determinism in the A-interpretation when one considers the dynamics of gauge-transformed gauge potentials in Hamiltonian phase space (i.e. a version of the well-known hole argument (cf. Earman and Norton 1987 and also Belot 2003). Healey (2001), on the other hand, argues for holonomies as the genuine entities in gauge theories simply because of Ockham's razor: due to its gauge invariance the $\mathrm{C}$-interpretation has no problem with questions about supposed privileged 
but empirically inaccessible gauges.

Secondly, there is an attempt by Nounou (2003) to give a fourth explanation of the $\mathrm{AB}$ effect which heavily leans on the fiber bundle formalism. This rich mathematical structure in its entirety is indispensable, Nounou claims, for a satisfactory explanation of the $\mathrm{AB}$ and, more generally, the topological effects in gauge theories. One may, however, ask what the real merit of this fourth explanation over the C-interpretation is. At the end of the day, holonomies are closely connected with the fiber bundle formalism - why should it be of more explanatory power then to "globally" point to the whole formalism instead of pointing to precisely the feature of that formalism - holonomies - which encode the crucial physical information? As far as our further considerations are concerned, this fourth explanation may rather be seen as intimately connected to the C-interpretation or as too vague to judge about its various locality constraints.

A further drawback of such a fourth explanation, in fact a general problem of an exaggerated emphasis of structures in physical theories, is to fall all to easy into a mysterious Platonism, where apparent "surplus structure," as Michael Redhead calls it, enters the stage of physical explanation. Redhead (2002) has discussed this within the context of a general analysis of gauge theories as one of the possible implications of the A-interpretation (cf. also the comment on Redhead by Drieschner, Eynck, Lyre 2002).

\subsection{Holonomies, non-separability and holism in gauge theories}

Particular attention should now be paid on the failure of separability as it occurs in the A- and C-interpretation, for their violation of separability seems to be of genuine gauge theoretic origin: in these two interpretations gauge theoretic entities as either the gauge potential or holonomies come into play, whereas the ontological furniture of the Binterpretation - field strengths - is still compatible with classical electromagnetism. What exactly is the reason for the non-separability in the A- and C-interpretation? As mentioned in the beginning of this section, the $\mathrm{AB}$ effect is only the prelude to a variety of topological effects in gauge theories as manifested by monopole or instanton solutions (cf. Fine and Fine 1997 for a philosophical discussion of gauge theoretic anomalies). But why call it a "prelude"?

The question which arises here is whether the $\mathrm{AB}$ effect is of geometrical or of topological origin. Note first that the holonomy is a non-integrable quantity, ${ }^{15}$ i.e. it does not only depend on the trajectory's starting and ending point, but is path dependent. A familiar example is the parallel transport of a vector in Riemannian spacetime along a closed curve. In the general case there will be a difference in angle between the initial and final vectors. Call this a geometrical holonomy. The quantum mechanical analogue is the well-known Berry or, more general, Aharonov-Anandan phase, where a quantum system acquires a phase shift merely because of its evolution through the Hamiltonian parameter

\footnotetext{
${ }^{15}$ Strictly speaking one must use the term anholonomy here, but the distinction between holonomic, integrable constraints and anholonomic, non-integrable ones in classical mechanics has not been taken over into the practice of differential geometry.
} 
space around some loop. Since this effect does depend on the particular path, Berry or Aharonov-Anandan phases are quite aptly characterized as geometric phases (cf. Shapere and Wilczek 1989 and Anandan 1992).

By way of contrast, a topological effect depends, in one way or the other, on the topology of the contributing space(s), i.e. the question whether one or more of the parameter, group or configuration spaces, which are involved in the effect, are non-simply connected. This is the case for genuine topological objects in gauge theories such as monopoles or instantons. These effects can be traced back to non-homotopic and, hence, non-trivial mappings $\mathbb{X} \rightarrow G$ of the configuration space onto the gauge group. E.g. in the case of $S U(2)$ instantons one considers $\mathbb{S}^{3} \rightarrow S U(2)$ and, thus, the non-triviality of the homotopy group $\pi_{3}\left(\mathbb{S}^{3}\right)=\mathbb{Z}$ (cf. Ryder 1996, chap. 10).

Insofar as holonomic effects of quantum phases are concerned, it is therefore, by analogy, useful to distinguish between geometric and topological phases (as Batterman 2003 has also pointed out). Now, in the AB case the holonomy is path dependent only in the sense that the solenoid has to be enclosed, but the particular shape of the curve is not essential. Moreover, since the electron can for all practical purposes be considered as perfectly shielded from the interior region of the solenoid, the configuration space of the electron can be modelled as $\mathbb{S}^{1}$ and is insofar doubly connected (all paths homotopic to $\mathbb{S}^{1}$ fall into the same topological equivalence class). Ryder (1996, p. 104) analyses the $\mathrm{AB}$ effect in this spirit as a genuine topological effect based on non-trivial mappings $\mathbb{S}^{1} \rightarrow U(1)$

However, as Brown and Pooley (2001) have recently pointed out, there is a proper classical analogue of the AB effect in Weylian spacetime known as the "second clock effect." In an experimental setting analogous to the AB setting one considers the transport of a measure of length in Weylian spacetime around the solenoid and will in general observe a length shift (Einstein in his early objection against Weyl's theory considered an analogous clock transport and pointed out that we do not observe such a supposed dependence of clock rates from their history). The classical Weylian AB effect does not fall under Ryder's topological analysis - for in this case the gauge group is just $\mathbb{R}$. And so, although the $\mathrm{AB}$ effect does not depend on the particular path, it still is a geometric effect, since only the fact counts that the curvature is non-vanishing in some (confined) region. ${ }^{16}$

What is, however, common to both genuine topological effects in gauge theories and the $\mathrm{AB}$ effect is their failure of separability. In all of these cases a non-separability results in the sense that the properties of the underlying loop holonomies do not supervene on the intrinsic properties of partitions of the loops. For instance, in the case of the AB

\footnotetext{
${ }^{16}$ In this sense the notion of "topological non-separability," as introduced in Eynck, Lyre and Rummell (forthcoming), should be dismissed. Nevertheless one must be careful with analogies to the original AB effect: As, for instance, Anandan (1993) and also Healey (this volume) point out, a decisive difference between phases in gravitational gauge theories (where one has to integrate over the connection of a suitable spacetime group such as $S O(1,3)$ or $\mathbb{R}^{1,3}$ ) and phases in quantum gauge theories such as the Dirac-Maxwell theory and Yang-Mill is that in the former cases the phase also has a value on open curves and not only as a holonomy.
} 
effect, the phase $\Delta \chi=q \oint_{\mathcal{C}} \vec{A} d \vec{r}$ cannot be derived from intrinsic properties either of the spacetime points or little pieces of the loop $\mathcal{C}$.

Moreover, we may very well distinguish this generic non-separability of the Cinterpretation from the well-known quantum theoretical cases. This becomes clear if we recall the above definitions of the two related notions of non-separability and holism. Quantum theory exhibits a general form of holism where no restriction of the domain of the system to its spatial or spatiotemporal support exists. By way of contrast, the failure of separability in gauge theories corresponds to spatiotemporal holism only as a particular variant or sub-concept of holism in general. Whether or not of course this distinction between non-separability and holism holds, remains at first a mere matter of terminology, nevertheless a fruitful distinction between cases of holism in quantum theory as opposed to gauge theories can be made.

\section{Structuralism in gauge theories}

“... realist about what? Is it the entities, the abstract structural relations, the fundamental laws or what? My own view is that the best candidate for what is 'true' about a physical theory is the abstract structural aspect. The main reason for this is that there is significantly greater continuity in structural aspects than there is in ontology, and one of the principal arguments against realism is the abrupt about-turns in ontological commitment even in the development of classical physics. Of course the structural aspects also change, but there is a more obvious sense in which one structure grows out of another in a natural development or generalization, so that a cumulative model of scientific progress is much easier to maintain."

Michael Redhead (1999)

\subsection{Why Structural Realism?}

Scientific realism is the view that our best current scientific theories are true and that their theoretical terms genuinely refer. Structural realism - a particular version of scientific realism - is further on the view that we ought to believe in the structural content of our best scientific theories only and that such structures refer. Why should one adopt such a view?

In the debate about scientific realism mainly two prominent arguments give rise to a sceptic and anti-realistic attitude: the pessimistic meta-induction and the underdetermination argument. The pessimistic meta-induction, as most forcefully argued by Larry 
Laudan (1981), and, similar in kind, the whole strand of Kuhnian incommensurability worries remind us of the fact that in earlier times there had been many empirically successful theories whose theoretical terms are not referring by our modern standards and that, today, we have no reason to believe that we are in any better situation. Thus, by induction over the history of science the prospects of realism are poor. Besides such historical arguments, theory underdetermination threatens the realist's supposed belief in a distinguished ontology with a systematic attack: Because of the seemingly surplus of the theoretical parts in our theories over the pure observational parts, there is no warrant, the underdetermination argument tells us, for the belief that a given body of empirical evidence can be satisfied by only one theory (and, hence, ontology) - even in the ultimate case of having all observational data at one's disposal.

On the other hand there is a forceful argument in favor of a realistic attitude - the wellknown no-miracles argument, which states the obvious: only realism offers an explanation for the remarkable success of science, which otherwise turns out as a sheer miracle. In the tension of these pros and cons, structural realism - a position already fashionable among neo-empiricists such as Russell and Carnap as well as the late Quine, among neo-Kantians like Cassirer, a whole strand of early 20th century physicists such as Poincaré, Eddington, Weyl and Wigner and philosophers of physics like Weizscker-was revived by John Worrall (1989) to avoid, on the one hand, the anti-realistic force of the pessimistic meta-induction and of theory underdetermination without making the success of science a miracle. The impossible becomes possible because structural realists do not commit themselves to an ontology of spatiotemporally fixed and object-like entities, such that worries about the empirical underdetermination of the object content of our best theories as well as about discontinuous shifts in theoretical entities in the course of scientific progress do not apply. What does, however, remain invariant or shows much more historical continuity, structural realists assert, is the structural content of scientific theories. In Worrall's words: structural realism offers "the best of both worlds."

Structural realism, as James Ladyman (1998) has pointed out, comes in two variants: an epistemic and an ontic form. And many if not most of the traditional structuralists should perhaps rather be considered as advocates of the more modest, epistemic variantfor they do not altogether dismiss the object-like entities, but merely consider them as epistemically inaccessible. By way of contrast, the radical ontic form of structural realism states that structure is all there is to the world. I shall not worry about the question what the ambiguous term "structure" could possibly all mean, since here we focus on physics and may therefore interpret the notion structure mainly in a mathematical sense (problems may arise if we ask for structural realism in cases of less mathematically formalized sciences such as biology, geology or the like). Examples for structural content in physical theories are state spaces, algebras of observables, the light-cone structure, fiber bundles and, last but not least, symmetry groups.

Within recent years now, the debate about structural realism, which before was preoccupied by arguments from the philosophy of science, receives reflourished interest from the philosophy of physics (cf. Redhead above and Cao, French, Ladyman and Saunders in 
a recent issue of Synthese, Vol. 136, 2003). French and Ladyman (2003) see direct support for structural realism - in their view even in its ontic form - in the metaphysical struggles about individuality in quantum theory as well as general relativity. Steven French (1998) particularly has stressed the point that in quantum theory we have the choice either to give up the notion of individual objects - the standard view about permutation symmetry of many particle states - or to introduce transcendental thisness or something similar to retain the metaphysical concept of individuality. The analogue of this problem in general relativity can be seen in the debate about the individuality of spacetime-points and connected questions about general covariance and the notorious hole argument (cf. most recently Stachel 2002). This underdetermination of metaphysics itself by physics, French argues, lends strong support to an ontic view of structural realism.

I shall not deal with this last, perhaps controversial issue, but rather my aim in the remaining part of this paper is to offer a third support leg for structural realism from the ontology of gauge theories and, particularly, $U(1)$ gauge theory-over and above the so far in the literature considered arguments from quantum and relativity theory. This shall now be laid out.

\subsection{Gauge Theoretic Structural Realism}

Four central arguments may be invoked for supporting structural realism from our gauge theoretic analysis. They follow from (i) the group-theoretic constitution of objects, (ii) the historical origin of $U(1)$ gauge theory, (iii) the ontological underdetermination of gauge theories, and (iv) the gauge theoretic holism. Let's consider them one after the other.

(i) Group-theoretic constitution of objects. In modern physics, objects are most generally defined as representations of symmetry groups, where the symmetries reflect spatiotemporal, i.e. external, and internal degrees of freedom as well as permutation invariance. Wigner's seminal analysis bears early witness of this circumstance. For the particular case of our Standard Model's elementary particles the relevant groups are the Poincaré group and the electroweak and chromodynamic gauge groups $S U(2)_{L} \times U(1)_{Y}$ and $S U(3)_{C}$. The group-theoretic constitution of objects is therefore not a peculiar feature of gauge theories alone, it certainly belongs to the overall tenor of modern physics (cf. Mittelstaedt 1994 and Castellani 1998). Since, however, internal degrees of freedom are described by gauge theories, we may list it as an argument here as well.

Technically speaking the set of states of an object forms its state space, which can be described as the representation space of a symmetry group. Hence, states transform into each other according to the action of the group on the specific representation. Elementary systems are described as irreducible representations where no further decomposition into invariant subspaces is possible. In $S U(n)$ gauge theories with principal bundle structure $\mathbb{P}(\mathcal{M}, S U(n))$ the matter field Dirac spinors are described as sections in associated vector bundles $\mathbb{E}\left(\mathcal{M}, \mathbb{C}^{n}\right)$ such that they constitute fundamental spinor representations of $S U(n)$. 
All this supports structural realism mainly because of two reasons. Firstly, a group theoretic definition of an object takes the group structure as primarily given, group representations are then construed from this structure and have a mere derivative status. Secondly, and more importantly, the group theoretic definition leads to object classes only, not to individual entities. Objects are merely defined as members of equivalence classes under symmetry transformations which leave certain object properties invariant. These properties, reflected by the Casimir operators of the group, are the remaining permanent or intrinsic properties of modern physics such as mass, spin and the various charges of the gauge interactions. It is, however, not possible to individuate objects from those, as we might also say, "structural" properties and this already undermines the traditional idea of objects as individuals.

Of course, this group-theoretic argument does not mean that we may dispense with the notion of objects as per se existing entities altogether, let alone the question, what kind of individuality those objects may have. In fact, a group-theoretic account of objects applies both to classical and quantum theory (we may, for instance, consider particles in classical mechanics as representations of the Galilean group). Therefore, and as already mentioned, structural realists like French expand on the issue of permutation symmetry in quantum theory and its impact on the (non-) individuality of quantum objects (French 1998, cf. also French and Rickles 2003). Moreover, John Stachel (2002) concluded that there is a close analogy between permutation invariance in quantum theory and the diffeomorphism invariance in general relativity by considering both cases within the general class of permutable theories, where the entities of such theories are only defined by the relational predicate structures of those theories, which must therefore be regarded as metaphysically primary.

While the group-theoretic constitution of objects in non-permutable theories might be considered as only an alternative to an account based on individual objects, in permutable theories such a description becomes mandatory. For there we only have access to the objects as members of equivalence classes under those symmetry transformations which leave the physical properties invariant. This is the situation in modern quantum gauge theories with regard to the field quanta of the fundamental matter and gauge fields and in gravitational gauge theories with regard to spacetime points.

(ii) Historical origin of gauge theories. Structural realism asserts the retention of mathematical structure through theory change. Worrall, and before him Poincaré already, have noticed that in the development from Fresnel's optics to Maxwell's electrodynamics the structure of the central equations remains invariant. Let us consider the next historical step: We already mentioned the gauge freedom present in Maxwell's theory and as expressed in the transformations (3). These gauge transformations form a group isomorphic to $\mathbb{R}$. When combined with Dirac's matter field theory, Maxwell's theory becomes embedded into the wider framework of the Dirac-Maxwell gauge theory or, once quantized fields are considered, into quantum electrodynamics. Within the larger mathematical fiber bundle structure of QED, the original gauge group of Maxwell's theory 
isomorphic to $\mathbb{R}$ turns out as the Lie algebra (in which the gauge connection takes its values) to the corresponding Lie gauge group $U(1)$.

Whereas, from the viewpoint of an ontology of objects, many of the entities in the transition of Maxwell's theory to QED have changed (Maxwell himself, for instance, still had the picture of an ether and neither potentials nor holonomies play a physical role in classical electrodynamics), there is a considerable element of retention of group structure and its embedding into a larger framework which makes the scientific progress much less discontinuous as it looks on the level of objects. Furthermore, the generalization from gauge group $U(1)$ to higher unitary groups or even gauge groups of gravity is - from a mathematical point of view - simply achieved by retaining the entire fiber bundle structure of a gauge theory and merely changing the particular groups. This simple recipe has led to the most powerful development in the history of physics: the advent of the Standard Model.

Certainly, however, the Standard Model is neither experimentally entirely vindicated (we have no evidence about the Higgs field), nor would one expect it to be the last word in physics (given the ad hoc-character of many of its conceptual premisses and the missing reason for the values of about 19 of its parameters). The above historical remarks do therefore shed only some light into the direction of the structural realist's conception of historical change in science, but do of course stand or fall with future scientific developments. However, developments in quantum gravity do already quite heavily lean on gauge theoretic concepts - and this may further endorse the structuralist's view (cf. also footnote 17 and Dorato 2000 for a discussion of quantum gravity in the light of structural realism).

(iii) Ontological underdetermination of gauge theories. As the analysis in section 2 shows, the object content of gauge theories, i.e. its ontology in terms of object-like entities, seems indeed to be strongly underdetermined. The interpretability of gauge theories either in terms of field strengths, potentials or holonomies clearly undermines a classical entity realism, whereas by the same time the structural content - the symmetry group and fiber bundle structure - remains the same in all three rivaling interpretations.

One may stress the fact that indeed the general issue of theory underdetermination is controversial within the philosophy of science. This is not to a slight extent due to the fact that there exist only a few, if at all, remarkable examples - even more remarkably, only a few authors seem to be really worried about this and try to come up with more convincing case studies. From this perspective the gauge theoretic ABC-example may at least be counted as a "more convincing" and "remarkable" case, but a generally critical stance on theory underdetermination leads us to the last point in the list of arguments.

(iv) Gauge theoretic holism. Among the three rivaling interpretations of gauge theories the C-interpretation is perhaps the one with the least ontological costs (cf. Belot 1998, 2003, Healey 2001 and Lyre 2001 for views in favor of holonomies as genuine entities). Reasons for this simply are that, first, realists can hardly be satisfied by the gauge 
dependence of entities as imminent in the A-interpretation and that, second, for the enterprise of physics in toto, it might perhaps be easier to cope with non-separability than with action-at-a-distance. Doesn't this undermine the underdetermination claim in the preceding point (iii) then? One might, on the one hand, still insist that our metaphysical premisses about locality cannot themselves be tested empirically - and this is what is at stake in the above underdetermination scenario. It is, on the other hand, most certainly true that our preference for an interpretation of a piece of physics over the other is guided by a variety of meta-criteria such as coherence, simplicity, scientific unity and the like. And it is the C-interpretation which seems to bear the biggest potential for further developments of physics. ${ }^{17}$

Put in a nutshell: on the basis of our present physical knowledge the above underdetermination scenario seems to stand firm - although meta-criteria already point into the direction of the C-interpretation. Future evidence may however more and more favor one over the other interpretations and let the claimed underdetermination become far less plausible and convincing. ${ }^{18}$ And of course, such further evidence will also imply the strengthening or weakening of the different meta-criteria involved. Whether developments like this may lead to further scepticism against the idea of underdetermination in general, cannot and must not be decided here, since, interestingly, in the case at hand structural realists may take advantage of both scenarios: either in the usual way that underdetermination undermines entity realism or in the sense of the spatiotemporal holism involved in the (A- and) C-interpretation.

This latter point means that, on the supposition that the C-interpretation is the one with the least ontological costs (i.e. the most consistent and coherent with the rest of physics in the future), our empirical world on its bottom level appears deeply nonseparable or spatiotemporally holistic. Admittedly, we may still consider holonomies as object-like entities, but to such an extent that our notion of an object becomes highly abstract. Traditional ontology, which thinks of objects as being spatiotemporally fixed, is clearly undermined.

\section{Concluding remarks}

A - perhaps necessary - repetition first: Our critical remarks in section 1 concerning the gauge principle and its much restricted physical meaning could perhaps give some reader the impression - though clearly wrong - that gauge theories are in total physically void. Instead, what has been tried to show in section 1 was that, first, gauge transformations

\footnotetext{
17 The so-called loop approach is chiefly studied because of its supposed prospects in solving problems of quantum gravity (cf. Gambini and Pullin 1996).

${ }^{18}$ Most recently, John Norton (2003) has argued that theory underdetermination should perhaps rather be seen as a variant of the induction problem - and the foregoing formulation indicates this as well. But despite such skeptical views on the issue of underdetermination, supposed cases of underdetermination might then have their practical value as indicators of incomplete scientific knowledge and the need to focus our scientific efforts on them (cf. Lyre and Eynck 2003).
} 
are not observable and that, in turn, the introduction of an interaction cannot be enforced by the gauge principle's postulate of local gauge invariance. But of course, and as already pointed out at the end of section 1, the characterization of, for instance, quantum electrodynamics as a $U(1)$ gauge theory is a physically meaningful statement. Prima facie it means that the curvature tensor of $U(1)$ can be given a physical interpretation in terms of an empirically vindicated interaction field strength. In the light of our former analysis this raises two points now: First, since our ontological analysis sheds doubt on the interpretation of gauge theories in terms of specific entities such as the field strengths (giving them a structurally derived and, thus, at most secondary status), we should rather rephrase the statement about the physical content of a gauge theory such that it is the group structure - as mathematically expressed in terms of the invariance under local $U(1)$ of the full dynamics represented by Lagrangian (6) - which is the holder of the physical meaning. Second, the deeper understanding of gauge theories in terms of their underlying principle(s) is - at least in my view - still an open issue. This issue, however, has no bearing on the discussion about the prospects of structural realism and may thus be skipped here. ${ }^{19}$

Now: do gauge theories - and in particular the $U(1)$ gauge theory - support structural realism? We have seen that the traditional picture of spatiotemporally fixed objectlike entities is undermined by the ontology of gauge theories in various ways and that main problems with traditional scientific realism - pessimistic meta-induction and theory underdetermination - can be softened by a commitment to the structural content of gauge theories, in particular to the gauge symmetry groups. We are obviously on much safer ground if we restrict ourselves to an ontology of gauge structures only, and from this perspective structural realism most certainly gains further credence from the framework of modern gauge theories.

On the other hand: from the tension between the third (iii) and fourth (iv) argument in the preceding section one may get the idea that the notions of object and structure do represent only extremal points of a whole continuum. More abstract and holistic notions of entities such as non-separable holonomies lie somewhere in-between. But this then looks like a return of usual entity realism just with more abstract means - and one must indeed admit that such an option cannot yet be dismissed on the basis of our gauge theoretic analysis so far.

However, putting more emphasis on the appearance of rather abstract entities instead of arguing in favor of structural realism on the basis of rejecting the pessimistic metainduction and the thread of underdetermination shouldn't necessarily be considered a disadvantage. On the contrary, a philosophical view such as structural realism gains by far more credence if supported by arguments from science directly than by mere indirect and notoriously debatable considerations of the philosophy of science. This is the difference in style between the Worrall-type of arguments in favor of structural realism and the Frenchtype of arguments - on the basis of the ontology of quantum theory — or the Stachel-type-

\footnotetext{
${ }^{19} \mathrm{An}$, admittedly controversial, attempt has been made to capture the main physical content of gauge theories in terms of a gauge theoretic generalization of the equivalence principle (Lyre 2000).
} 
on the basis of general relativity.

When we stick to more abstract and non-local entities we do not dismiss the notion of objects altogether. This may help to understand an otherwise troublesome objection against structural realism: What exactly is it that we measure in our laboratories at CERN or elsewhere when all there is in the world, fundamentally, is just "group structure?" What kind of measurability could we assign to this asserted structure if not by means of ever so abstract objects? The epistemic structural realist might have an answer at hand: objects exist but we don't have a firm epistemic grip on them. That's why we get lost in underdetermination scenarios and the like. Nevertheless the "real" objects are still the vehicles of our access to the bottom level structures.

For the radical ontic structural realist, on the other hand, the question seems to provide a serious problem. And even more so: the main problem with the notion of structure itself and our very metaphysical understanding of it is that it seems impossible to grasp the idea of relations without relata - but this is where the radical ontic version apparently leads us to.

A third "intermediate" possibility would be to stick with more abstract but still measurable or, as some authors like to phrase it, "structural" objects - and here non-separable holonomies turn out a suitable case at hand. Thus the question is indeed not so much whether we should dismiss the objects or relata altogether but whether they finally will be defined any other than in mere relational or structural terms.

Of course, the drawback of this option seems to be that if we still believe in-ever so abstract - objects the problems imposed by the pessimistic meta-induction or underdetermination are still not overcome. But again: underdetermination is a controversial issue in itself and might perhaps not be considered such a strong anti-realistic argument anyhow. And secondly: arguing against the pessimistic meta-induction on the basis of a still open theory - the Standard Model - can hardly lead to a line of argument which is full-proof. So even if the analysis of gauge theories given in this paper might in partspoints (ii) and (iii) in 3.2-be read as a Worrall-type support of structural realism, a more sustained conclusion should perhaps rather be seen in the development of modern physics into more abstract - here spatiotemporally holistic - entities in accordance with an intermediate version of structural realism - less radical than the ontic version but more directly supported from science than the epistemic one.

\section{Acknowledgements}

I am most grateful to both the anonymous referees and the editors of this journal for their entirely justified demands for clarification on several points. One report in particular had been very thorough and quite helpful. 


\section{References}

Aharonov, Y. and Bohm, D. (1959). Significance of electromagnetic potentials in the quantum theory. Physical Review, 115(3): 485-491.

Aitchison, I. J. and Hey, A. J. G. (1982). Gauge Theories in Particle Physics - A Practical Introduction. Hilger, Bristol.

Anandan, J. (1992). The geometric phase. Nature, 360: 307-313.

Anandan, J. (1993). Remarks concerning the geometries of gravity and gauge fields. In Hu, B. L., Ryan, M. P., and Vishveshwara, C. V., editors, Directions in General Relativity (Vol. 1). Cambridge University Press, Cambridge.

Auyang, S. Y. (1995). How is Quantum Field Theory Possible? Oxford University Press, New York.

Batterman, R. (2003). Falling cats, parallel parking, and polarized light. Studies in History and Philosophy of Modern Physics, 34 (4): 527-557.

Belot, G. (1998). Understanding electromagnetism. The British Journal for the Philosophy of Science, 49: 531-555.

Belot, G. (2003). Symmetry and gauge freedom. Studies in History and Philosophy of Modern Physics, 34: 189-225.

Belot, G. and Earman, J. (2001). Pre-socratic quantum gravity. In Callender and Huggett (2001).

Brading, K. and Brown, H. R. (2003). Noether's theorems. In Brading and Castellani (2003).

Brading, K. and Brown, H. R. (forthcoming). Are gauge symmetry transformations observable? The British Journal for the Philosophy of Science.

Brading, K. and Castellani, E., editors (2003). Symmetries in Physics: Philosophical Reflections. Cambridge University Press, Cambridge.

Brown, H. R. (1999). Aspects of objectivity in quantum mechanics. In Butterfield, J., and Pagonis, C., editors. From Physics to Philosophy. Cambridge University Press, Cambridge.

Brown, H. R. and Pooley, O. (2001). The origin of the spacetime metric: Bell's 'Lorentzian pedagogy' and its significance in general relativity. In Callender and Huggett (2001).

Callender, C. and Huggett, N., editors (2001). Physics meets Philosophy at the Planck Scale. Cambridge University Press, Cambridge. 
Cao, T. Y. (1997). Conceptual Developments of 20th Century Field Theories. Cambridge University Press, Cambridge.

Castellani, E. (1998a). Galilean particles: An example of constitution of objects. In Castellani (1998).

Castellani, E., editor (1998b). Interpreting Bodies: Classical and Quantum Objects in Modern Physics. Princeton University Press, Princeton.

DeWitt, B. S. (1962). Quantum theory without electromagnetic potentials. Physical Review, 125(6): 2189-2191.

Dorato, M. (2000). Substantivalism, relationism and structural spacetime realism. Foundations of Physics, 30(10): 1605-1628.

Drechsler, W. and Mayer, M. E. (1977). Fiber Bundle Techniques in Gauge Theories. Lecture Notes in Physics 67. Springer, Berlin.

Drieschner, M., Eynck, T. O., and Lyre, H. (2002). Comment on Redhead: The interpretation of gauge symmetry. In Kuhlmann, Lyre, Wayne (2002).

Earman, J. and Norton, J. (1987). What price spacetime substantivalism? The hole story. The British Journal for the Philosophy of Science, 83: 515-525.

Earman, J. (2002). Gauge matters. Philosophy of Science, 69(3): S209-S220.

Earman, J. (2003). Tracking down gauge: An ode to the constrained Hamiltonian formalism. In Brading and Castellani (2003).

Esfeld, M. (2001). Holism in Philosophy of Mind and Philosophy of Physics. Synthese Library No. 298. Kluwer, Dordrecht.

Eynck, T. O., Lyre, H., and Rummell, N. v. (forthcoming). A versus B! Topological nonseparability and the Aharonov-Bohm effect. (E-print PhilSci-Archive 404)

Feynman, R. P. (1963). The Feynman Lectures on Physics. Addison-Wesley, Reading, Mass.

Fine, D. and Fine, A. (1997). Gauge theory, anomalies and global geometry: The interplay of physics and mathematics. Studies in History and Philosophy of Modern Physics, 28(3): 307-323.

French, S. (1998). On the withering away of physical objects. In Castellani (1998).

French, S. and Ladyman, J. (2003). Remodelling structural realism: Quantum physics and the metaphysics of structure. Synthese, 136: 31-56.

French, S. and Rickles, D. (2003). Understanding permutation symmetry. In Brading and Castellani (2003). 
Gambini, R. and Pullin, J. (1996). Loops, Knots, Gauge Theories and Quantum Gravity. Cambridge University Press, Cambridge.

Healey, R. (1991). Holism and nonseparability. Journal of Philosophy, 88: 393-421.

Healey, R. (1997). Nonlocality and the Aharonov-Bohm effect. Philosophy of Science, 64: $18-41$.

Healey, R. (1999). Quantum analogies: A reply to Maudlin. Philosophy of Science, 66: 440-447.

Healey, R. (2001). On the reality of gauge potentials. Philosophy of Science, 68(4): $432-455$.

Healey, R. (this volume). Gauge theory and holisms.

Henneaux, M. and Teitelboim, C. (1992). Quantization of Gauge Systems. Princeton University Press, Princeton.

Hooft, G. t. (1980). Gauge theories of the forces between elementary particles. Scientific American, 242(6): 104-138.

Howard, D. (1985). Einstein on locality and separability. Studies in History and Philosophy of Science, 16: 171-201.

Kosso, P. (2000). The empirical status of symmetries in physics. The British Journal for the Philosophy of Science, 51: 81-98.

Kuhlmann, M., Lyre, H., and Wayne, A., editors (2002). Ontological Aspects of Quantum Field Theory. World Scientific, Singapore.

Ladyman, J. (1998). What is structural realism? Studies in History and Philosophy of Science, 29(3): 409-424.

Laudan, L. (1981). A confutation of convergent realism. Philosophy of Science, 48: $19-49$.

Leeds, S. (1999). Gauges: Aharonov, Bohm, Yang, Healey. Philosophy of Science, 66: 606-627.

Liu, C. (1994). The Aharonov-Bohm effect and the reality of wave packets. The British Journal for the Philosophy of Science, 45: 977-1000.

Liu, C. (2001). Gauge gravity and the unification of natural forces. (E-print PhilSciArchive 364).

Loll, R. (1994). The loop formulation of gauge theory and gravity. In Baez, J. C., editor, Knots and Quantum Gravity. Oxford University Press, Oxford. 
Lyre, H. (2000). A generalized equivalence principle. International Journal of Modern Physics D, 9(6): 633-647.

Lyre, H. (2001). The principles of gauging. Philosophy of Science, 68(3): S371-S381.

Lyre, H. and Eynck, T. O. (2003). Curve it, gauge it or leave it? Practical underdetermination in gravitational theories. Journal for General Philosophy of Science, 34(2): 277-303.

Martin, C. (2002). Gauge principles, gauge arguments and the logic of nature. Philosophy of Science, 69(3): S221-S234.

Martin, C. (2003). Continuous symmetry and the foundations of physics. In Brading and Castellani (2003).

Maudlin, T. (1998). Discussion: Healey on the Aharonov-Bohm effect. Philosophy of Science, 65: 361-368.

Mittelstaedt, P. (1994). Constitution of objects in classical mechanics and in quantum mechanics. International Journal of Theoretical Physics, 34(8): 1615-1626.

Nakahara, M. (1990). Geometry, Topology and Physics. IOP Publishing, Bristol.

Norton, J. D. (2003). Must evidence underdetermine theory? (E-print PhilSci-Archive $1257)$.

Nounou, A. (2003). A fourth way to the Aharonov-Bohm effect. In Brading and Castellani (2003).

Papineau, D., editor (1996). The Philosophy of Science. Oxford University Press, Oxford.

Pauli, W. (1941). Relativistic field theories of elementary particles. Reviews of Modern Physics, 13: 203-232.

Peshkin, M. A. and Tonomura, A. (1989). The Aharonov-Bohm Effect. Lecture Notes in Physics 340. Springer, Berlin.

O'Raifeartaigh, L. (1995). The Dawning of Gauge Theory. Princeton University Press, Princeton.

Redhead, M. (1999). Quantum field theory and the philosopher. In Cao, T. Y., editor. Conceptual Foundations of Quantum Field Theory. Cambridge University Press, Cambridge.

Redhead, M. (2002). The interpretation of gauge symmetry. In Kuhlmann, Lyre, Wayne (2002).

Ryder, L. H. (1996). Quantum Field Theory. Cambridge University Press, Cambridge. (Second edition). 
Saunders, S. (2003). Critical notice: Tian Yu Caos "The conceptual development of 20th century field theories". Synthese, 136: 79-105.

Schutz, B. F. (1980). Geometrical Methods of Mathematical Physics. Cambridge University Press, Cambridge.

Shapere, A. and Wilczek, F., editors (1989). Geometric Phases in Physics. World Scientific, Singapore.

Stachel, J. (2002). "The relations between things" versus "The things between relations": The deeper meaning of the hole argument. In Malament, D. B., editor. Reading Natural Philosophy: Essays in the History and Philosophy of Science and Mathematics. Open Court, La Salle, Illinois.

Teller, P. (1986). Relational holism and quantum mechanics. The British Journal for the Philosophy of Science, 37:71-81.

Teller, P. (1999). Some questions about the QED coupling constant. Lecture at the Int. Conf. on "Ontological Aspects of Quantum Field Theory," Bielefeld, October 1999.

Teller, P. (2000). The gauge argument. Philosophy of Science, 67(3): S466-S481.

Tonomura, A. (1998). The Quantum World Unveiled by Electron Waves. World Scientific, Singapore.

Trautman, A. (1980). Fiber bundles, gauge fields, and gravitation. In Held, A., editor. General Relativity and Gravitation: One Hundred Years after the Birth of Albert Einstein. Plenum Press, New York.

Worrall, J. (1989). Structural realism: The best of both worlds? Dialectica, 43: 99-124. 